\title{
Risk of pregnancy complications and adverse birth outcomes after maternal A(H1N1)pdm09 influenza: a Norwegian population-based cohort study
}

Ida Laake ${ }^{1 *}$ D, Gro Tunheim ${ }^{2,3}$, Anna Hayman Robertson ${ }^{1}$, Olav Hungnes ${ }^{4}$, Kristian Waalen $^{4}$, Siri E. Håberg ${ }^{5}$, Siri Mjaaland ${ }^{2,3}$ and Lill Trogstad ${ }^{1}$

\begin{abstract}
Background: The effects of maternal influenza infection on the fetus remain unclear. We studied mild influenza and influenza antibodies in relation to birth weight and risks of pre-eclampsia, preterm birth (PTB), and small for gestational age (SGA) birth among the unvaccinated participants in the Norwegian Influenza Pregnancy Cohort.

Methods: Pregnant women attending a routine ultrasound were recruited from four hospitals in Norway shortly after the 2009 A(H1N1) pandemic. The present study was restricted to unvaccinated participants who were pregnant during the pandemic. Information on the participants was obtained through questionnaires and linkage with national registries. Maternal blood samples were collected at delivery. Women with laboratory-confirmed A(H1N1)pdm09 influenza, a clinical diagnosis of influenza, or self-reported influenza during the pandemic were classified as having had influenza. A(H1N1)pdm09-specific antibodies in serum were detected with the hemagglutinationinhibition assay. Detection of antibodies was considered an indicator of infection during the pandemic in the unvaccinated participants. Odds ratios were estimated with logistic regression. Quantile regression was used to estimate differences in the distribution of birth weight.
\end{abstract}

Results: Among the 1258 women included in this study, there were 37 cases of pre-eclampsia, 41 births were PTB, and 103 births were SGA. 226 women (18.0\%) had influenza during the pandemic. The majority of cases did not receive medical care, and only a small proportion (1.3\%) of the cases were hospitalized. Thus, the cases consisted primarily of women with mild illness. No significant associations between influenza and risk of pre-eclampsia, PTB, or SGA birth were observed. Detection of A(H1N1)pdm09-specific antibodies was associated with a lower 10th percentile of birth weight, $\beta=-159 \mathrm{~g}(95 \% \mathrm{Cl}-309$, - 9).

Conclusions: Mild influenza illness during pregnancy was not associated with increased risk of pre-eclampsia, PTB or SGA birth. However, influenza infection during pregnancy may reduce the birth weight of the smallest children.

Keywords: Influenza, Pandemic, A(H1N1)pdm09, Antibodies, Pregnancy, Birth outcomes

\footnotetext{
* Correspondence: ida.laake@fhi.no

${ }^{1}$ Department of Infectious Disease Epidemiology and Modelling, Norwegian

Institute of Public Health, Oslo, Norway

Full list of author information is available at the end of the article
}

(c) The Author(s). 2018 Open Access This article is distributed under the terms of the Creative Commons Attribution 4.0 International License (http://creativecommons.org/licenses/by/4.0/), which permits unrestricted use, distribution, and reproduction in any medium, provided you give appropriate credit to the original author(s) and the source, provide a link to the Creative Commons license, and indicate if changes were made. The Creative Commons Public Domain Dedication waiver (http://creativecommons.org/publicdomain/zero/1.0/) applies to the data made available in this article, unless otherwise stated. 


\section{Background}

Influenza may cause severe illness and death in pregnant women. During the $\mathrm{A}(\mathrm{H} 1 \mathrm{~N} 1)$ influenza pandemic in 2009, pregnant women with influenza had higher risk of hospitalization than non-pregnant individuals with influenza [1, 2]. Less is known about the effects of maternal influenza infection on the fetus. Maternal infections may increase the risk of pre-eclampsia [3], a major cause of intrauterine growth restriction and preterm birth (PTB), but there are few studies on influenza and risk of pre-eclampsia [4-7]. High rates of PTB were observed among pregnant women hospitalized with influenza during the 2009 pandemic [8-10], but these studies did not include a comparison group of pregnant women without influenza. Several other studies have included both pregnant women with and without influenza such that appropriate comparisons of the risk of adverse birth outcomes like PTB, low birth weight, or fetal death could be made $[7,11-18]$. However, the number of studies on each outcome is limited, and results are inconsistent. Thus, in a recent systematic review, no firm conclusions could be drawn regarding maternal influenza in relation to the main outcomes PTB, small for gestational age (SGA) birth, and fetal death [19]. Moreover, in most studies, the influenza cases consist of women who sought medical care, thus women with mild infection are unlikely to be included among the cases. Since a substantial proportion of infected individuals develop mild illness or remain asymptomatic [20], studies on the impact of mild influenza infection on pregnancy complications and outcomes are needed.

The Norwegian Influenza Pregnancy Cohort (NorFlu) Study is a population-based cohort of women who were pregnant during the pandemic in 2009. Using this cohort, we studied maternal influenza in relation to the risk of pre-eclampsia and adverse birth outcomes among the unvaccinated participants.

\section{Methods}

\section{The NorFlu study}

In Norway, the main wave of the pandemic occurred from October 1, 2009 to December 31, 2009 [15]. A vaccination campaign against $\mathrm{A}(\mathrm{H} 1 \mathrm{~N} 1) \mathrm{pdm} 09$ was started in mid-October 2009 [21]. The vaccine was recommended to all pregnant women in the second or third trimester.

The participants in the NorFlu Study were recruited from four hospitals (three in the Oslo-area and one in Bergen), where they had received the ultrasound examination offered to all pregnant women around pregnancy week 18. The ultrasound examination is usually performed at the hospital where the birth is planned. Women who had their last menstrual period between June 1, 2009 and December 1, 2009 were invited by mail to participate in the study. Recruitment took place during pregnancy week 28-40, from February 2010 to September 2010. Out of 5333 pregnant women who were invited, 3201 women (60.0\%) agreed to participate. The majority of the participants were pregnant in the first or second trimester during the main wave of the pandemic. Upon return of the informed consent form that was included with the invitation, the participants were sent two questionnaires, one covering influenza and influenza vaccination and one covering pregnancy and general health. Questionnaires were completed and returned prior to delivery. Blood samples were collected at delivery from a total of 2408 (75.2\%) of the participants. The protocol did not specify any exclusion criteria for blood sampling, but the capacity in the delivery unit may have prevented blood sample collection from some participants. In addition, a few women may have been unwilling to provide a blood sample. In June 2010, the influenza and vaccination questionnaire was sent to approximately 12,000 participants in another large pregnancy cohort, the Norwegian Mother and Child Cohort Study (MoBa) [22]. Women from the Oslo- or Bergen area who reported being pregnant during the past 12 months were subsequently invited to participate in the NorFlu Study. Of the 1769 invited women, 1291 gave their consent to participate. Blood samples could not be collected from these women.

The participants were linked to their records in national registries and databases by use of the unique identification number assigned to all residents of Norway. Information about the pregnancy, delivery, and infant was obtained from the Medical Birth Registry of Norway (MBRN). Records of vaccination against $\mathrm{A}(\mathrm{H} 1 \mathrm{~N} 1) \mathrm{pdm} 09$ influenza were obtained from the Norwegian Immunization Registry. Contacts with primary care physicians that resulted in a diagnosis of influenza (R-80, International Classification of Primary Care, Second edition) were obtained from the Directorate of Health's reimbursement database. Cases of laboratory-confirmed $\mathrm{A}(\mathrm{H} 1 \mathrm{~N} 1) \mathrm{pdm} 09$ influenza were obtained from the Norwegian Surveillance system for Communicable Diseases.

\section{Outcomes}

We studied the following outcomes: pre-eclampsia, PTB, SGA birth, and birth weight. Women were classified as having pre-eclampsia if mild pre-eclampsia, severe pre-eclampsia, or pre-eclampsia before week 34 had been reported to MBRN. PTB was defined as birth before 37 completed weeks of gestation. SGA birth was defined as birth weight $<10$ th percentile for gestational age and sex [23]. Few singletons born in Norway have birth weight $<2500 \mathrm{~g}$, the World Health Organization's definition of low birth weight. Therefore, birth weight was treated as a continuous variable. 


\section{Definition of exposures Influenza}

Women were classified as having had influenza during the pandemic if they were diagnosed with influenza by a primary care physician during the main wave of the pandemic (October 1, 2009 to December 31, 2009), had laboratory confirmed A(H1N1)pdm09 influenza, or reported having influenza-like illness in October, November, or December of 2009 on the questionnaire.

\section{Antibodies against a(H1N1)pdm09}

Sera were analyzed for antibodies against A(H1N1)pdm09 using the hemagglutination-inhibition (HI) assay in serial two-fold dilutions, starting at dilution 1:10 [24]. HI-titer was defined as the reciprocal of the highest dilution that produced complete inhibition in the assay. The women were classified as having HI-titer $\geq 10$ or HI-titer $=5$, corresponding to an undetectable level of antibodies. The $\mathrm{A}(\mathrm{H} 1 \mathrm{~N} 1) \mathrm{pdm} 09$ virus was antigenically very distinct from previous seasonal H1N1 viruses, and before the 2009 pandemic, the prevalence of antibodies against $\mathrm{A}(\mathrm{H} 1 \mathrm{~N} 1) \mathrm{pdm} 09$ was very low among Norwegian women of reproductive age [25]. Since the sera in our study were collected after the pandemic, we assume that the presence of antibodies against $\mathrm{A}(\mathrm{H} 1 \mathrm{~N} 1) \mathrm{pdm} 09$ is due to vaccination or influenza infection during the pandemic. Consequently, in unvaccinated women, detected antibodies indicate influenza infection during the pandemic.

\section{Other covariates}

Women who self-reported having one or more of the medical conditions that are considered risk factors for developing influenza complications (asthma, diabetes type 1 , diabetes type 2 , other lung diseases, obesity, cardiovascular disease, kidney disease, or impaired immune system) were considered part of an influenza risk group. Information on smoking during pregnancy was incomplete in both the general health questionnaire and the MBRN. Therefore, women were classified as smokers if they had smoked during pregnancy according to either of these sources. Start of pregnancy was set to 282 days prior to the ultrasound predicted date of birth, or the date of the last menstrual period if the ultrasound predicted date was missing.

\section{Study sample}

In total, 4492 pregnant women participated (Fig. 1). The current study was restricted to women not vaccinated against $\mathrm{A}(\mathrm{H} 1 \mathrm{~N} 1) \mathrm{pdm} 09$. We used the influenza questionnaire in addition to the immunization registry to determine vaccination status, since approximately $10 \%$ of those vaccinated against $\mathrm{A}(\mathrm{H} 1 \mathrm{~N} 1) \mathrm{pdm} 09$ during the pandemic were not registered in the immunization registry [26]. In total, 2738 vaccinated women were excluded
(Fig. 1). Furthermore, we excluded women who did not fill out the influenza questionnaire. Women who could not be linked to the MBRN were also excluded since the outcomes were not known for these women. Women with unknown pregnancy start and women who were not pregnant during the main wave of the pandemic were also excluded. Finally, we excluded women with multiple births, missing information on smoking and women who had influenza before pregnancy. In total, 1258 women were included in the analyses with influenza as the main exposure.

Blood samples were collected from 788 of the 1258 women. Of these, 12 women were missing HI-titers. The analyses with HI-titer as the main exposure were therefore limited to the remaining 776 women with available HI-titer measurements.

\section{Statistical analysis}

To examine influenza and HI-titer in relation to risk of pre-eclampsia, PTB, and SGA birth we used logistic regression to estimate odds ratios (ORs) with corresponding 95\% confidence intervals (CIs). We used quantile regression [27] to estimate the effects of influenza and HI-titer on birth weight. For binary exposure variables, the quantile regression coefficient, $\beta$, is the difference in a specific percentile of birth weight when comparing the exposed to the unexposed. Since we were mainly interested in the smallest children, we assessed the 10th percentile and the 50th percentile (median). The analyses were adjusted for maternal age at birth (continuous), parity (primiparous/multiparous), smoking during pregnancy (yes/no), and influenza risk group (yes/no). Since the blood samples were collected at delivery, several months after the pandemic, we also adjusted the analyses with HI-titer as the main exposure for time in days between birth and the pandemic (continuous), in order to account for possible waning of the HI-titers. In the subset of women with HI-titer, no women in the influenza risk group developed pre-eclampsia. Influenza risk group was therefore removed from the model when pre-eclampsia was the outcome and HI-titer the exposure. Since we could not determine exact time of exposure to $\mathrm{A}(\mathrm{H} 1 \mathrm{~N} 1) \mathrm{pdm} 09$ for women with HI-titer $\geq 10$, we performed additional analyses limited to women with pregnancy start before October 1, 2009, i.e. before the main wave of the pandemic, thus excluding women who could potentially have been exposed before conception.

\section{Results}

All births $(n=1258)$ took place in the period October 22, 2009 to October 13, 2010, but the majority (83.1\%) occurred between May 2010 and August 2010. 37 women developed pre-eclampsia. Furthermore, 41 births were PTB, and 103 births were SGA. Mean gestational 


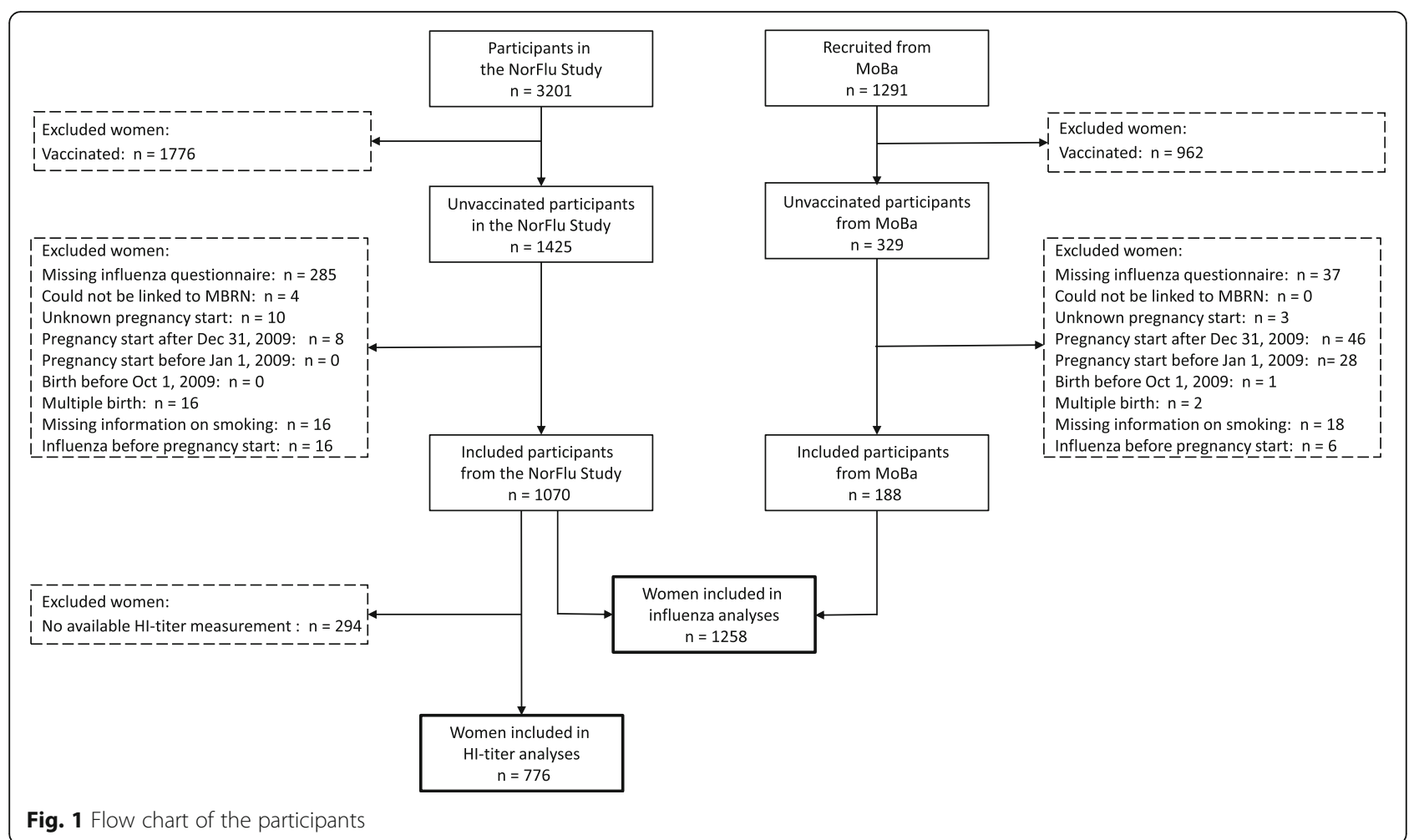

age was 281 days, and mean birth weight was 3587 g. 226 women $(18.0 \%)$ had influenza during the pandemic. Nearly half of the influenza cases (45.6\%) were medically attended, i.e. either diagnosed by a primary care physician or laboratory confirmed A(H1N1)pdm09 influenza (Table 1). The remaining 123 cases (54.4\%) were based solely on self-report. Antiviral medication was used by 35 $(15.5 \%)$ of the women with influenza. Only 3 women (1.3\% of cases) reported that they were hospitalized with influenza. Compared to women without influenza, women with influenza were more likely to belong to an influenza risk group, less likely to be primiparous, and less likely to have smoked during pregnancy (Table 2). In addition, pregnancy start was earlier among women with influenza.

\section{Influenza}

Using a wide definition of influenza (see above) that included both self-reported and medically attended cases, influenza during pregnancy was not significantly associated

Table 1 Description of influenza cases, $n=226$

\begin{tabular}{ll}
\hline Medically attended, $\mathrm{n}(\%)$ & $103(45.6)$ \\
Influenza diagnosis (R80) from primary health care, $\mathrm{n}(\%)^{\mathrm{a}}$ & $91(40.3)$ \\
${\text { Laboratory confirmed, } \mathrm{n}(\%)^{\mathrm{a}}}$ & $38(16.8)$ \\
Based on self-report, not medically attended, $\mathrm{n}(\%)$ & $123(54.4)$ \\
Antiviral medication, $\mathrm{n}(\%)$ & $35(15.5)$ \\
Hospitalized, $\mathrm{n}(\%)$ & $3(1.3)$ \\
\hline
\end{tabular}

${ }^{\mathrm{a}} 26$ cases were both diagnosed in primary health care and laboratory confirmed with risk of pre-eclampsia, PTB, or SGA birth among the women in our study: ORs were 1.44 (95\% CI $0.64,3.26), 0.77$ (95\% CI $0.32,1.88)$, and 1.35 (95\% CI $0.80,2.28$ ), respectively (Table 3 ). Furthermore, birth weight was similar for women with and without influenza, the difference in the 10th percentile was $22 \mathrm{~g}(95 \% \mathrm{CI}-130,174)$, and the difference in the median was $-4 \mathrm{~g}(95 \% \mathrm{CI}-74,67)$.

Table 2 Characteristics of study sample by influenza status

\begin{tabular}{|c|c|c|}
\hline & $\begin{array}{l}\text { Without } \\
\text { influenza, } \\
n=1032\end{array}$ & $\begin{array}{l}\text { With influenza, } \\
n=226\end{array}$ \\
\hline Influenza risk group (\%) & 5.6 & 13.3 \\
\hline Age of mother, years (mean) & 32.0 & 31.6 \\
\hline No previous births, (\%) & 46.7 & 37.6 \\
\hline Use of folic acid during pregnancy (\%) & 69.6 & 71.2 \\
\hline Smoking during pregnancy (\%) & 4.5 & 1.3 \\
\hline Date of pregnancy start (median) & Oct 02, 2009 & Sept 12, 2009 \\
\hline $\begin{array}{l}\text { Second or third trimester at start of } \\
\text { pandemic }(\%)^{\mathrm{a}}\end{array}$ & 5.6 & 8.4 \\
\hline $\begin{array}{l}\text { Days between end of pandemic and } \\
\text { birth (mean) })^{b}\end{array}$ & 186 & 170 \\
\hline Date of birth (median) & July 11, 2010 & June 21, 2010 \\
\hline $\begin{array}{l}\text { Recruited from the Norwegian Mother } \\
\text { and Child Cohort Study (\%) }\end{array}$ & 15.1 & 14.2 \\
\hline Blood sample collected at birth (\%) & 61.3 & 68.6 \\
\hline
\end{tabular}

aStart of pandemic defined as October 1, 2009

${ }^{\mathrm{b}}$ End of pandemic defined as December 31, 2009 
Table 3 Influenza in relation to risk of pre-eclampsia, preterm birth, small for gestational age birth, and birthweight

\begin{tabular}{|c|c|c|}
\hline & $\begin{array}{l}\text { Without influenza, } \\
n=1032\end{array}$ & $\begin{array}{l}\text { With influenza, } \\
n=226\end{array}$ \\
\hline \multicolumn{3}{|l|}{ Pre-eclampsia } \\
\hline Cases, n (\%) & $29(2.8)$ & $8(3.5)$ \\
\hline Crude OR (95\% Cl) & 1 (Ref) & $1.27(0.57,2.81)$ \\
\hline Adjusted OR $(95 \% \mathrm{Cl})^{\mathrm{a}}$ & 1 (Ref) & $1.44(0.64,3.26)$ \\
\hline \multicolumn{3}{|l|}{ Preterm birth } \\
\hline Cases, n (\%) & $35(3.4)$ & $6(2.7)$ \\
\hline Crude OR (95\% Cl) & 1 (Ref) & $0.78(0.32,1.87)$ \\
\hline Adjusted OR $(95 \% \mathrm{Cl})^{\mathrm{a}}$ & 1 (Ref) & $0.77(0.32,1.88)$ \\
\hline \multicolumn{3}{|l|}{ Small for gestational age birth } \\
\hline Cases, n (\%) & $82(7.9)$ & $21(9.3)$ \\
\hline Crude OR (95\% Cl) & 1 (Ref) & $1.19(0.72,1.96)$ \\
\hline Adjusted OR $(95 \% \mathrm{Cl})^{\mathrm{a}}$ & 1 (Ref) & $1.35(0.80,2.28)$ \\
\hline \multicolumn{3}{|l|}{ Birth weight } \\
\hline 10th percentile (g) & 2975 & 2898 \\
\hline $\begin{array}{l}\text { Difference in 10th percentile, } \\
\beta(95 \% \mathrm{Cl})^{b}\end{array}$ & 1 (Ref) & $22(-130,174)$ \\
\hline 50th percentile (g) & 3589 & 3614 \\
\hline $\begin{array}{l}\text { Difference in 50th percentile, } \\
\beta(95 \% \mathrm{Cl})^{b}\end{array}$ & 1 (Ref) & $-4(-74,67)$ \\
\hline
\end{tabular}

aLogistic regression adjusted for maternal age at birth (continuous), parity (primiparous/multiparous), smoking during pregnancy (yes/no), and influenza risk group (yes/no)

${ }^{\mathrm{b}}$ Quantile regression adjusted for maternal age at birth (continuous), parity (primiparous/multiparous), smoking during pregnancy (yes/no), and influenza risk group (yes/no)

\section{Antibodies against a(H1N1)pdm09}

In the subsample of 776 women with measured HI-titers, the prevalence of influenza (self-reported and medically attended cases combined) during the pandemic was almost three times higher among women with HI-titer $\geq 10$ than among women with HI-titer $=5$, i.e. those with an undetectable level of antibodies (Table 4). The two groups were similar in terms of age, parity, and time of pregnancy start.

We observed no significant associations between HI-titer and risk of pre-eclampsia, PTB, or SGA birth: ORs were 0.83 (95\% CI 0.29, 2.36), 1.26 (95\% CI 0.47, 3.40 ), and 1.30 (95\% CI 0.74, 2.81), respectively (Table 5). In contrast, we observed a close to significant difference $(p=0.055)$ in the 10th percentile of birth weight when comparing women with HI-titer $\geq 10$ to women with HI-titer $=5, \quad \beta=-123$ g $(95 \%$ CI $-248,2)$. Median birth weight was similar in the two groups, $\beta=-10 \mathrm{~g}(95 \% \mathrm{CI}-97,76)$.

In the subgroup of women with babies in the lowest decile of birthweight, gestational age was similar among those with HI-titer $=5$ and those with HI-titer $\geq 10$ (269 days vs 270 days). The prevalence of influenza in
Table 4 Characteristics of study sample by maternal HI-titer at delivery

\begin{tabular}{|c|c|c|}
\hline & $\begin{array}{l}\text { HI-titer }=5 \\
n=575\end{array}$ & $\begin{array}{l}\text { HI-titer } \geq 10 \\
n=201\end{array}$ \\
\hline Influenza (\%) & 13.2 & 37.8 \\
\hline Influenza risk group (\%) & 7.8 & 8.0 \\
\hline Age of mother, years (mean) & 32.0 & 31.7 \\
\hline No previous births (\%) & 53.6 & 48.8 \\
\hline Smoking during pregnancy (\%) & 3.3 & 5.0 \\
\hline Use of folic acid during pregnancy (\%) & 69.9 & 69.2 \\
\hline Date of pregnancy start (median) & Sept 29, 2009 & Sept 22, 2009 \\
\hline $\begin{array}{l}\text { Second or third trimester at start of } \\
\text { pandemic }(\%)^{\mathrm{a}}\end{array}$ & 2.8 & 2.0 \\
\hline $\begin{array}{l}\text { Days between end of pandemic and } \\
\text { birth (mean) }\end{array}$ & 188 & 184 \\
\hline Date of birth (median) & July 08, 2010 & July 03, 2010 \\
\hline
\end{tabular}

Table 5 HI-titer in relation to risk of pre-eclampsia, preterm birth, small for gestational age birth, and birth weight

\begin{tabular}{|c|c|c|}
\hline & $\begin{array}{l}\text { HI-titer }=5 \\
n=575\end{array}$ & $\begin{array}{l}\text { HI-titer } \geq 10 \\
n=201\end{array}$ \\
\hline \multicolumn{3}{|l|}{ Pre-eclampsia } \\
\hline Cases, n (\%) & $16(2.8)$ & $5(2.5)$ \\
\hline Crude OR (95\% Cl) & 1 (Ref) & $0.89(0.32,2.46)$ \\
\hline Adjusted OR $(95 \%$ CI) & 1 (Ref) & $0.83(0.29,2.36)$ \\
\hline
\end{tabular}

Preterm birth

$\begin{array}{lll}\text { Cases, n (\%) } & 13(2.3) & 6(3.0) \\ \text { Crude OR }(95 \% \mathrm{Cl}) & 1 \text { (Ref) } & 1.33(0.50,3.55) \\ \text { Adjusted OR }(95 \% \mathrm{Cl})^{\mathrm{a}} & 1 \text { (Ref) } & 1.26(0.47,3.40)\end{array}$

Small for gestational age birth

$\begin{array}{lll}\text { Cases, n (\%) } & 45(7.8) & 20(10.0) \\ \text { Crude OR }(95 \% \mathrm{Cl}) & 1 \text { (Ref) } & 1.30(0.75,2.26) \\ \text { Adjusted OR }(95 \% \mathrm{Cl})^{\mathrm{a}} & 1 \text { (Ref) } & 1.30(0.74,2.81)\end{array}$

Birth weight

$\begin{array}{lll}\text { 10th percentile }(\mathrm{g}) & 3000 & 2932 \\ \begin{array}{l}\text { Difference in 10th percentile, } \\ \beta(95 \% \mathrm{Cl})^{\mathrm{b}}\end{array} & 1 \text { (Ref) } & -123(-248,2) \\ \text { 50th percentile }(\mathrm{g}) & 3560 & 3580 \\ \begin{array}{l}\text { Difference in 50th percentile, } \\ \beta\left(95 \%()^{b}\right.\end{array} & 1 \text { (Ref) } & -10(-97,76)\end{array}$
$\beta(95 \% \mathrm{Cl})^{\mathrm{b}}$

${ }^{a}$ Logistic regression adjusted for maternal age at birth (continuous), parity (primiparous/multiparous), smoking during pregnancy (yes/no), and time in days between birth and pandemic (continuous). The model was also adjusted for influenza risk group (yes/no) except with pre-eclampsia as the outcome ${ }^{b}$ Quantile regression adjusted for maternal age at birth (continuous), parity (primiparous/multiparous), smoking during pregnancy (yes/no), influenza risk group (yes/no), and time in days between birth and pandemic (continuous) 
this subgroup was $20.0 \%$ for women with HI-titer $=5$ and $34.6 \%$ for women with HI-titer $\geq 10$. Compared to women with HI-titer $=5$, nearly twice as many women with $\mathrm{HI}$-titer $\geq 10$ belonged to an influenza risk group (10.0\% vs $19.2 \%)$.

Among women with pregnancy start before October 1, $2009(n=411)$, HI-titer was not significantly associated with risk of pre-eclampsia, OR $=0.98$ ( $95 \%$ CI 0.18 , 5.46), or with risk of PTB, OR $=1.30$ (95\% CI 0.31, 5.41) (Table 6). Women with HI-titer $\geq 10$ had higher risk of SGA birth than women with HI-titer $=5$, but this was not significant, OR $=1.75$ (95\% CI 0.77, 3.97). However, the 10th percentile of birth weight was significantly lower for women with HI-titer $\geq 10$ than women with HI-titer $=5, \beta=-159$ g (95\% CI $-309,-9)$. Median birth weight was slightly lower for women with HI-titer $\geq 10, \beta=-31$ g $(95 \% \mathrm{CI}-158,96)$.

\section{Discussion}

In this study, we investigated potentially adverse effects of maternal influenza on birth outcomes in a cohort of women that were pregnant during the influenza

Table 6 HI-titer in relation to risk of pre-eclampsia, preterm birth, small for gestational age birth, and birth weight in women with pregnancy start before October 1, 2009

\begin{tabular}{|c|c|c|}
\hline & $\begin{array}{l}\text { Hl-titer }=5 \\
n=293\end{array}$ & $\begin{array}{l}\text { Hl-titer } \geq 10 \\
n=118\end{array}$ \\
\hline \multicolumn{3}{|l|}{ Pre-eclampsia } \\
\hline Cases, n (\%) & $6(2.0)$ & $2(1.7)$ \\
\hline Crude OR (95\% CI) & 1 (Ref) & $0.82(0.16,4.15)$ \\
\hline Adjusted OR $(95 \% \mathrm{Cl})^{\mathrm{a}}$ & 1 (Ref) & $0.98(0.18,5.46)$ \\
\hline \multicolumn{3}{|l|}{ Preterm birth } \\
\hline Cases, n (\%) & $7(2.4)$ & $3(2.5)$ \\
\hline Crude OR (95\% CI) & 1 (Ref) & $1.07(0.27,4.19)$ \\
\hline Adjusted OR $(95 \% \mathrm{Cl})^{\mathrm{a}}$ & 1 (Ref) & $1.30(0.31,5.41)$ \\
\hline \multicolumn{3}{|l|}{ Small for gestational age birth } \\
\hline Cases, n (\%) & $17(5.8)$ & $11(9.3)$ \\
\hline Crude OR (95\% Cl) & 1 (Ref) & $1.67(0.76,3.68)$ \\
\hline Adjusted OR (95\% Cl) & 1 (Ref) & $1.75(0.77,3.97)$ \\
\hline \multicolumn{3}{|l|}{ Birth weight } \\
\hline 10th percentile (g) & 3070 & 2940 \\
\hline $\begin{array}{l}\text { Difference in 10th percentile, } \\
\beta(95 \% \mathrm{Cl})^{\mathrm{b}}\end{array}$ & 1 (Ref) & $-159(-309,-9)$ \\
\hline 50th percentile (g) & 3560 & 3610 \\
\hline $\begin{array}{l}\text { Difference in 50th percentile, } \\
\beta(95 \% \mathrm{Cl})^{b}\end{array}$ & 1 (Ref) & $-31(-158,96)$ \\
\hline \multicolumn{3}{|c|}{$\begin{array}{l}{ }^{a} \text { Logistic regression adjusted for maternal age at birth (continuous), parity } \\
\text { (primiparous/multiparous), smoking during pregnancy (yes/no), and time in } \\
\text { days between birth and pandemic (continuous). The model was also adjusted } \\
\text { for influenza risk group (yes/no) except with pre-eclampsia as the outcome } \\
{ }^{\mathrm{b}} \text { Quantile regression adjusted for maternal age at birth (continuous), parity } \\
\text { (primiparous/multiparous), smoking during pregnancy (yes/no), influenza risk } \\
\text { group (yes/no), and time in days between birth and pandemic (continuous) }\end{array}$} \\
\hline
\end{tabular}

pandemic in 2009. The influenza cases consisted primarily of women with mild illness. The majority of cases were not medically attended, and only a small proportion of the cases were hospitalized. Neither influenza in pregnancy nor detection of maternal antibodies against $\mathrm{A}(\mathrm{H} 1 \mathrm{~N} 1) \mathrm{pdm} 09$ at the time of delivery were significantly associated with risk of pre-eclampsia, PTB, or SGA birth. However, detection of antibodies was associated with more than $100 \mathrm{~g}$ lower 10th percentiles of birth weight, both overall and among women with pregnancy start before the main wave of the pandemic.

The NorFlu Study is a population-based cohort, providing a unique opportunity to study the impact of mild influenza in pregnancy. Extensive information was gathered on the participants. Through linkage with the MBRN, a national registry with data of high quality, we had complete and accurate information on the outcomes we studied. In order to capture both the milder cases of influenza and those that were medically attended, we combined information from different sources. Moreover, blood samples from a large number of women were tested for antibodies against $\mathrm{A}(\mathrm{H} 1 \mathrm{~N} 1) \mathrm{pdm} 09$, an objective indicator of infection in unvaccinated individuals that is not influenced by recall. Serology may detect both symptomatic and asymptomatic infections, and serological surveys have been used to estimate the cumulative incidence of $\mathrm{A}(\mathrm{H} 1 \mathrm{~N} 1)$ pdm09 infection [28].

The main limitation of our study is the possible misclassification of the exposures. Due to limited capacity during the pandemic, laboratory testing of suspected $\mathrm{A}(\mathrm{H} 1 \mathrm{~N} 1)$ pdm09 influenza cases was restricted [21]. Less than $1 / 5$ of the cases in our study, and only about $1 / 3$ of the medically attended cases, were laboratory confirmed. Approximately half of the influenza cases were based on self-reported illness. However, the participants in our study were pregnant at the time of the pandemic, so we would expect them to recall illness more accurately than the general population. Moreover, since the questionnaire was completed prior to birth, misclassification does not depend on the outcomes we studied. Furthermore, we required that all the influenza cases, both the self-reported and the medically attended, were ill during the main wave of the pandemic, thereby reducing the likelihood that their illness was caused by infectious agents other than $\mathrm{A}(\mathrm{H} 1 \mathrm{~N} 1) \mathrm{pdm} 09$, since this was by far the dominating respiratory virus accounting for influenza-like illness during the main wave of the pandemic in Norway [29].

Given the unpredictable nature of both influenza pandemic occurrence and pregnancy, we did not have the opportunity to collect any pre-pandemic samples. Thus, we could not identify the infected individuals according to an increase in $\mathrm{HI}$-titer. Influenza seropositivity is commonly defined as HI-titer $\geq 40$ [28], which is 
associated with a $50 \%$ reduced risk of influenza infection [30]. However, a substantial proportion of individuals infected during the pandemic did not become seropositive [31, 32], and the use of HI-titer of 40 as a threshold has been found to lead to an underestimate of the cumulative incidence of influenza infection during the pandemic [31]. Moreover, the prevalence of antibodies against $\mathrm{A}(\mathrm{H} 1 \mathrm{~N} 1) \mathrm{pdm} 09$ among Norwegian women aged 20 to 40 years was very low before the 2009 pandemic [25]. Therefore, detection of antibodies (HI-titer $\geq 10$ ) after the pandemic is probably a good proxy for infection in the unvaccinated women in our cohort. However, a small proportion in our cohort may have had cross-reactive antibodies against $\mathrm{A}(\mathrm{H} 1 \mathrm{~N} 1) \mathrm{pdm} 09$ from previous influenza infections. On the other hand, due to HI-titer waning [33], some of the infected women may have had undetectable levels of antibodies as the mean time between the pandemic and blood sampling was 6 months. However, this time interval was adjusted for in the analyses.

Few of the women in our study experienced adverse birth outcomes. We observed 41 cases of PTB among the 1258 women included in the analyses, corresponding to a rate of $3.3 \%$. Among all the women who participated in the study, the rate of singleton PTB was $3.1 \%$, which is lower than the average rate of $5.3 \%$ observed among live-born singletons in Norway in 2008 [34]. The lower rate may be explained by the NorFlu participants being more health conscious than the general population. This is supported by the high proportion of participants using folic acid during pregnancy (according to their record in the Medical Birth Registry) compared to all women in Norway giving birth in 2010 (71.5\% vs. 27.0\%) [35]. The participants in the NorFlu study may not be representative of the general population, but this does not necessarily result in biased effect estimates. A study on self-selection in MoBa found lower prevalence of several risk factors, pregnancy complications, and adverse birth outcomes among participants than among all women giving birth in Norway [36]. However, this study found no evidence of bias in exposure-outcome associations. Recruitment to NorFlu was based on the experience from MoBa and followed the same procedures, thus we expect this to be true for the NorFlu study also.

Even though the information on the outcomes was almost complete in this study, and only a handful of the participants were lost to follow-up, we did not have complete information on the exposures. More than $10 \%$ of the unvaccinated women did not return the influenza questionnaire and were therefore excluded. Among these women, the rate of singleton PTB was $6.0 \%$, considerably higher than among the women included in the study. Furthermore, women without a blood sample who were excluded from the analyses with HI-titer as the main exposure, had a higher rate of singleton PTB than women included in these analyses (4.6\% vs $2.4 \%)$. This indicates that the included participants tend to be healthier than the excluded participants. It is possible that influenza infection has a more harmful effect on birth outcomes for less healthy individuals with certain underlying conditions. In that case, the selection will probably have resulted in an underestimation of the effects of influenza during pregnancy.

In previous studies, mild $\mathrm{A}(\mathrm{H} 1 \mathrm{~N} 1) \mathrm{pdm} 09$ influenza during pregnancy was not associated with mean birth weight $[7,16]$ or increased risk of PTB [14-16], SGA birth $[7,14,16]$, or birth weight $<2500 \mathrm{~g}[7,14-16]$. This is in correspondence with our results. Risks of hospitalization and death due to influenza are highest in the third trimester [37], but whether the timing of influenza exposure during pregnancy is of importance in relation to birth outcomes is not clear. In a recent study from Canada, no increased risk of PTB was observed for women with medically attended pandemic influenza in their first or second trimester [18]. Influenza in the third trimester was associated with significantly increased risk of PTB, but only in the subgroup of women belonging to an influenza risk group. Since recruitment to our study started in February 2010, the majority of the participants were in their first trimester during the main wave of the pandemic. Thus, we could not study whether the impact of influenza differs with trimester of exposure.

Influenza and detection of $\mathrm{A}(\mathrm{H} 1 \mathrm{~N} 1)$ pdm09 antibodies were not significantly associated with risk of pre-eclampsia in our study. As far as we are aware, only two studies have previously investigated $\mathrm{A}(\mathrm{H} 1 \mathrm{~N} 1) \mathrm{pdm} 09$ influenza during pregnancy and risk of pre-eclampsia $[6,7]$. In both studies, women with $\mathrm{A}(\mathrm{H} 1 \mathrm{~N} 1) \mathrm{pdm} 09$ influenza were compared to women with suspected influenza who tested negative for $\mathrm{A}(\mathrm{H} 1 \mathrm{~N} 1) \mathrm{pdm} 09$. In accordance with our results, no significant differences in the proportion with pre-eclampsia were observed in these studies.

Only a few, mainly older, studies have used serology to identify women infected with influenza during pregnancy, thus also capturing milder and asymptomatic influenza cases [38-41]. When comparing infected and uninfected mothers, none of these studies found significant differences in mean birth weight [39-41] or proportion with low birth weight [38]. Detection of antibodies was not associated with median birth weight in our study. However, the 10th percentile of birth weight was more than 100 g lower for women with HI-titer $\geq 10$ than women with undetectable levels of antibodies. In the subgroup with birth weight in the lowest decile, HI-titer was not associated with gestational age. Thus, the difference in birth weight does not seem to be a 
result of lower gestational age among those with HI-titer $\geq 10$. Possibly, influenza infection may have a direct effect on intrauterine growth. This is supported by randomized clinical trials (RCTs) on maternal influenza vaccination [42, 43]. In RCTs, a higher risk of adverse birth outcomes among unvaccinated women can be attributed to the higher incidence of influenza infection. In line with our results, the RCTs found that women who received influenza vaccine had lower risk of low birth weight compared to women in the control group, although a significant difference in mean birth weight was also observed. In our study, a high proportion of women with HI-titer $\geq 10$ and birth weight in the lowest decile belonged to an influenza risk group (19.2\%). This may indicate that influenza infection has a stronger effect on birth weight for mothers with underlying conditions. Whether the offspring of less healthy pregnant women are more susceptible to the harmful effects of influenza infection should be further studied.

\section{Conclusions}

Very few women in this population-based cohort of women who were pregnant during the 2009 pandemic had severe influenza. Furthermore, we found little evidence that mild influenza during pregnancy is associated with increased risk of pre-eclampsia, PTB or SGA birth. However, our findings may suggest that even mild or asymptomatic influenza infection during pregnancy may reduce the birth weight of the smallest children.

\section{Abbreviations \\ Cl: Confidence interval; MBRN: The Medical Birth Registry of Norway; NorFlu: The Norwegian Influenza Pregnancy Cohort; OR: Odds ratio; PTB: Preterm birth; SGA: Small for gestational age}

\section{Acknowledgements}

The authors wish to thank the staff at Oslo University Hospital, Bærum Hospital and Haukeland Hospital for recruitment of pregnant women and blood collection. We also thank all the members of the NorFlu study group at the Norwegian Institute of Public Health and the Influenza Centre at the University of Bergen for the planning, administration of data collection, data linkage, handling and storage of biological samples, and laboratory analyses. We are also grateful to all the women who participated in the NorFlu study.

\section{Funding}

The study was funded by the Research Council of Norway (grant number 221122). The funder had no role in the design of the study, data collection, analysis, interpretation of the data, or in preparation of the manuscript.

\section{Availability of data and materials}

The data analyzed during this study consist of sensitive information on an individual level. Due to protection of privacy and restrictions from the Norwegian Data Inspectorate and the Regional Committee for Medical and Health Research Ethics, the data are not publicly available. However, it is possible to apply for access to data from the NorFlu study for scientific purposes (https://www.fhi.no/en/studies/norflu/applying-for-data-from-norflu).

\section{Authors' contributions}

LT, SEH, and SM initiated and designed the NorFlu Study. LT conceived of the present study. $\mathrm{OH}$ and $\mathrm{KW}$ were responsible for the hemagglutinationinhibition assay of the sera. IL performed the statistical analyses and drafted the manuscript. GT, AHR, SM, and LT contributed to the interpretation of the data and writing of the manuscript. All authors revised the manuscript critically for important intellectual content. All authors read and approved the final manuscript.

Ethics approval and consent to participate

The study was approved by the Regional Committee for Medical and Health Research Ethics, Southeast Norway on December 22, 2009 (reference number 2009/2165). Written informed consent was obtained from all participants.

\section{Consent for publication}

Not applicable

\section{Competing interests}

The authors declare that they have no competing interests.

\section{Publisher's Note}

Springer Nature remains neutral with regard to jurisdictional claims in published maps and institutional affiliations.

\section{Author details}

'Department of Infectious Disease Epidemiology and Modelling, Norwegian Institute of Public Health, Oslo, Norway. ${ }^{2}$ Department of Infectious Disease Immunology, Norwegian Institute of Public Health, Oslo, Norway. ${ }^{3}$ K.G. Jebsen Center for Influenza Vaccine Research, University of Oslo, Oslo, Norway. ${ }^{4}$ Department of Influenza, Norwegian Institute of Public Health, Oslo, Norway. ${ }^{5}$ Division for Mental and Physical Health, Norwegian Institute of Public Health, Oslo, Norway.

Received: 13 February 2018 Accepted: 5 October 2018

Published online: 22 October 2018

\section{References}

1. Van Kerkhove MD, Vandemaele KA, Shinde V, Jaramillo-Gutierrez G, Koukounari A, Donnelly CA, et al. Risk factors for severe outcomes following 2009 influenza a (H1N1) infection: a global pooled analysis. PLoS Med. 2011;8:e1001053.

2. Mertz D, Geraci J, Winkup J, Gessner BD, Ortiz JR, Loeb M. Pregnancy as a risk factor for severe outcomes from influenza virus infection: a systematic review and meta-analysis of observational studies. Vaccine. 2017;35:521-8.

3. Rustveld LO, Kelsey SF, Sharma R. Association between maternal infections and preeclampsia: a systematic review of epidemiologic studies. Matern Child Health J. 2008;12:223-42.

4. Acs N, Banhidy F, Puho E, Czeizel AE. Pregnancy complications and delivery outcomes of pregnant women with influenza. J Matern Fetal Neonatal Med. 2006:19:135-40.

5. Rogers VL, Sheffield JS, Roberts SW, Mclntire DD, Luby JP, Trevino S, et al. Presentation of seasonal influenza a in pregnancy: 2003-2004 influenza season. Obstet Gynecol. 2010;115:924-9.

6. Mendez-Figueroa H, Raker C, Anderson BL. Neonatal characteristics and outcomes of pregnancies complicated by influenza infection during the 2009 pandemic. Am J Obstet Gynecol. 2011;204:S58-63.

7. Nieto-Pascual L, Arjona-Berral JE, Marin-Martin EM, Munoz-Gomariz E, llich I, Castelo-Branco C. Early prophylactic treatment in pregnant women during the 2009-2010 H1N1 pandemic: obstetric and neonatal outcomes. J Obstet Gynaecol. 2013;33:128-34.

8. Louie JK, Acosta M, Jamieson DJ, Honein MA. Severe 2009 H1N1 influenza in pregnant and postpartum women in California. N Engl J Med. 2010;362: 27-35.

9. The ANZIC Influenza Investigators and Australasian Maternity Outcomes Surveillance System. Critical illness due to 2009 a/H1N1 influenza in pregnant and postpartum women: population based cohort study. BMJ. 2010;340:c1279.

10. Siston AM, Rasmussen SA, Honein MA, Fry AM, Seib K, Callaghan WM, et al. Pandemic 2009 influenza a(H1N1) virus illness among pregnant women in the United States. JAMA. 2010;303:1517-25.

11. Cox S, Posner SF, McPheeters M, Jamieson DJ, Kourtis AP, Meikle S. Hospitalizations with respiratory illness among pregnant women during influenza season. Obstet Gynecol. 2006;107:1315-22. 
12. McNeil SA, Dodds LA, Fell DB, Allen VM, Halperin BA, Steinhoff MC, et al. Effect of respiratory hospitalization during pregnancy on infant outcomes. Am J Obstet Gynecol. 2011;204:S54-7.

13. Pierce M, Kurinczuk JJ, Spark P, Brocklehurst P, Knight M. Perinatal outcomes after maternal 2009/H1N1 infection: national cohort study. BMJ. 2011;342:d3214.

14. Hansen C, Desai S, Bredfeldt C, Cheetham C, Gallagher M, Li DK, et al. A large, population-based study of 2009 pandemic influenza a virus subtype H1N1 infection diagnosis during pregnancy and outcomes for mothers and neonates. J Infect Dis. 2012;206:1260-8.

15. Håberg SE, Trogstad L, Gunnes N, Wilcox AJ, Gjessing HK, Samuelsen SO, et al. Risk of fetal death after pandemic influenza virus infection or vaccination. $N$ Engl J Med. 2013;368:333-40.

16. Naresh A, Fisher BM, Hoppe KK, Catov J, Xu J, Hart J, et al. A multicenter cohort study of pregnancy outcomes among women with laboratoryconfirmed H1N1 influenza. J Perinatol. 2013;33:939-43.

17. Doyle TJ, Goodin K, Hamilton JJ. Maternal and neonatal outcomes among pregnant women with 2009 pandemic influenza a(H1N1) illness in Florida, 2009-2010: a population-based cohort study. PLoS One. 2013;8:e79040

18. Fell DB, Platt RW, Basso O, Wilson K, Kaufman JS, Buckeridge DL, et al. The relationship between 2009 pandemic H1N1 influenza during pregnancy and preterm birth: a population-based cohort study. Epidemiology. 2018;29:107-16.

19. Fell DB, Savitz DA, Kramer MS, Gessner BD, Katz MA, Knight M, et al. Maternal influenza and birth outcomes: systematic review of comparative studies. BJOG. 2017;124:48-59.

20. Hayward AC, Fragaszy EB, Bermingham A, Wang L, Copas A, Edmunds WJ, et al. Comparative community burden and severity of seasonal and pandemic influenza: results of the flu watch cohort study. Lancet Respir Med. 2014;2:445-54

21. Guzman Herrador BR, Aavitsland P, Feiring B, Riise Bergsaker MA, Borgen K. Usefulness of health registries when estimating vaccine effectiveness during the influenza a(H1N1)pdm09 pandemic in Norway. BMC Infect Dis. 2012;12:63.

22. Magnus P, Irgens LM, Haug K, Nystad W, Skjærven R, Stoltenberg C, et al. Cohort profile: the Norwegian mother and child cohort study (MoBa). Int J Epidemiol. 2006:35:1146-50.

23. Skjærven R, Gjessing HK, Bakketeig LS. Birthweight by gestational age in Norway. Acta Obstet Gynecol Scand. 2000;79:440-9.

24. Waalen K, Kilander A, Dudman SG, Ramos-Ocao R, Hungnes O. Agedependent prevalence of antibodies cross-reactive to the influenza a(H3N2) variant virus in sera collected in Norway in 2011. Euro Surveill. 2012;17(19): 20170.

25. Waalen K, Kilander A, Dudman SG, Krogh GH, Aune T, Hungnes O. High prevalence of antibodies to the 2009 pandemic influenza a $(\mathrm{H} 1 \mathrm{~N} 1)$ virus in the Norwegian population following a major epidemic and a large vaccination campaign in autumn 2009. Euro Surveill. 2010;15(31):19633.

26. Trogstad L, Ung G, Hagerup-Jenssen M, Cappelen I, Haugen IL, Feiring B. The Norwegian immunisation register-SYSVAK. Euro Surveill. 2012;17(16): 20147.

27. Koenker R, Hallock KF. Quantile regression. J Econ Perspect. 2001;15:143-56.

28. Van Kerkhove MD, Hirve S, Koukounari A, Mounts AW. Estimating agespecific cumulative incidence for the 2009 influenza pandemic: a metaanalysis of a(H1N1)pdm09 serological studies from 19 countries. Influenza Other Respir Viruses. 2013;7:872-86

29. The Norwegian Institute of Public Health. Influensaovervåking 2009-10, uke 52 og 53 [Influenza surveillance 2009-10, week 52 and 53]. https://www.fhi. no/historisk-arkiv/artikler/influensa2/influensarapporter/influensaovervaking2009-10-uke-52-/. Accessed 30 Nov 2017. (In Norwegian).

30. Hobson D, Curry RL, Beare AS, Ward-Gardner A. The role of serum haemagglutination-inhibiting antibody in protection against challenge infection with influenza A2 and B viruses. J Hyg (Lond). 1972;70:767-77.

31. Zhao X, Siegel K, Chen MI, Cook AR. Rethinking thresholds for serological evidence of influenza virus infection. Influenza Other Respir Viruses. 2017;11: 202-10.

32. Veguilla V, Hancock K, Schiffer J, Gargiullo P, Lu X, Aranio D, et al. Sensitivity and specificity of serologic assays for detection of human infection with 2009 pandemic H1N1 virus in U.S. populations. J Clin Microbiol. 2011;49: 2210-5

33. Hsu JP, Zhao X, Chen MI, Cook AR, Lee V, Lim WY, et al. Rate of decline of antibody titers to pandemic influenza a (H1N1-2009) by hemagglutination inhibition and virus microneutralization assays in a cohort of seroconverting adults in Singapore. BMC Infect Dis. 2014;14:414.
34. Zeitlin J, Szamotulska K, Drewniak N, Mohangoo AD, Chalmers J, Sakkeus L, et al. Preterm birth time trends in Europe: a study of 19 countries. BJOG. 2013;120:1356-65

35. The Norwegian Institute of Public Health. Medical Birth Registry of Norway statistics bank. http://statistikkbank.fhi.no/mfr/. Accessed 16 Aug 2018.

36. Nilsen RM, Vollset SE, Gjessing HK, Skjaerven R, Melve KK, Schreuder $P$, et al. Self-selection and bias in a large prospective pregnancy cohort in Norway. Paediatr Perinat Epidemiol. 2009;23:597-608.

37. Mertz D, Kim TH, Johnstone J, Lam PP, Science M, Kuster SP, et al. Populations at risk for severe or complicated influenza illness: systematic review and metaanalysis. BMJ. 2013;347:f5061.

38. Wilson MG, Stein AM. Teratogenic effects of asian influenza. An extended study. JAMA. 1969;210:336-7.

39. Korones SB, Todaro J, Roane JA, Sever JL. Maternal virus infection after the first trimester of pregnancy and status of offspring to 4 years of age in a predominantly negro population. J Pediatr. 1970;77:245-51.

40. Griffiths PD, Ronalds CJ, Heath RB. A prospective study of influenza infections during pregnancy. J Epidemiol Community Health. 1980;34:124-8.

41. Irving $W L$, James DK, Stephenson T, Laing $P$, Jameson C, Oxford JS, et al. Influenza virus infection in the second and third trimesters of pregnancy: a clinical and seroepidemiological study. BJOG. 2000;107:1282-9.

42. Steinhoff MC, Omer SB, Roy E, El Arifeen S, Raqib R, Dodd C, et al. Neonatal outcomes after influenza immunization during pregnancy: a randomized controlled trial. CMAJ. 2012;184:645-53.

43. Steinhoff MC, Katz J, Englund JA, Khatry SK, Shrestha L, Kuypers J, et al. Yearround influenza immunisation during pregnancy in Nepal: a phase 4 randomised, placebo-controlled trial. Lancet Infect Dis. 2017;17:981-9.

\section{Ready to submit your research? Choose BMC and benefit from:}

- fast, convenient online submission

- thorough peer review by experienced researchers in your field

- rapid publication on acceptance

- support for research data, including large and complex data types

- gold Open Access which fosters wider collaboration and increased citations

- maximum visibility for your research: over $100 \mathrm{M}$ website views per year

At BMC, research is always in progress.

Learn more biomedcentral.com/submissions 